\title{
Air pollution reduction measures in the Sydney GMR using marginal abatement cost curves
}

\author{
M. Davies ${ }^{1}$, B. Kearney ${ }^{2} \&$ A. Morison ${ }^{3}$ \\ ${ }^{1}$ Air Quality and Greenhouse Gas Emissions, SKM, Newcastle, Australia \\ ${ }^{2}$ Strategic Consulting, SKM, Sydney, Australia \\ ${ }^{3}$ Air Policy and Programs Unit, Office of Environment and Heritage, \\ NSW Department of Premier and Cabinet, Australia
}

\begin{abstract}
New South Wales, Australia (NSW) is committed to achieving air quality goals set in 1998 by the National Environment Protection Measure (Ambient Air Quality). The Office of Environment and Heritage (OEH) has estimated that a $25 \%$ reduction in anthropogenic emissions of volatile organic compounds (VOC) and oxides of nitrogen (NOX) from 2003 levels is required to meet ozone goals in Sydney.

The OEH engaged Sinclair Knight Merz (SKM) to identify and analyse emission abatement initiatives across the Greater Metropolitan Region (GMR). SKM developed a Marginal Abatement Cost Curve (MACC) model to assess the economic, environmental and social impacts as well as technical feasibility of each identified initiative.

The curves identify potential sets of strategies that could be applied to achieve target emission reductions at the least estimated cost, and provide a guide to prioritising potential actions for further investigation. Prior to implementation, further full analysis of potential actions is required to determine the actual costs and emission abatement potential.

Key components of the study included: a methodology for developing MACCs for measures to reduce anthropogenic NOX, VOCs and PM10 emissions in the GMR; a list of identified emission abatement initiatives; assessment of each initiative to determine important costing information and emissions abatement likely to be achieved by each initiative; cost curves for NOX, VOCs and PM10; and optimisation of the curves, considering the multipollutant framework of emissions abatement.
\end{abstract}


The reference case used for the MACC modelling represents emissions for the GMR. Emissions were projected from 2003 to 2031, assuming prior trends in emissions profiles and drivers continue, adjusted only for existing or highly likely policies or industry projects.

Separate pollutant MACCs were developed for the GMR, Sydney and Wollongong. A total of 35 abatement initiatives were completed for the GMR with a smaller subset of these applicable in the Sydney and Wollongong regions. Keywords: cost curve, air management.

\section{Introduction}

NSW is committed to achieving air quality goals set in 1998 by the National Environment Protection Measure (Ambient Air Quality). The Office of Environment and Heritage $(\mathrm{OEH})$ has estimated that a reduction in anthropogenic emissions of volatile organic compounds (VOC) and oxides of nitrogen (NOX) by $25 \%$ from 2003 levels is required to meet ozone goals in Sydney [1].

$\mathrm{OEH}$ engaged Sinclair Knight Merz (SKM) to undertake a study which identified and analysed a range of emission abatement initiatives across the Greater Metropolitan Region (GMR) and sub-regions of NSW. SKM developed a Marginal Abatement Cost Curve (MACC) model to assist in assessing the practicability of each identified initiative from a number of perspectives including economic, environmental and social impacts as well as technical feasibility. Separate MACC curves were developed for each of the substances considered in this study: VOC, NOX and particulates (PM10). The curves show the cost and abatement quantity from a range of potential initiatives to reduce emissions in the NSW GMR. Health benefits were not included in the assessment.

The curves identify potential sets of strategies that could be applied to achieve emission reductions at the least estimated cost, and are intended to provide a guide to prioritising potential actions for further investigation. Costs and emission abatement estimates for actions on which the curves are based are indicative and not always readily compared across actions, given that they are drawn from a range of studies and jurisdictions.

\section{Study components}

Key components of the study included:

- A methodology for developing MACCs for measures to reduce anthropogenic $\mathrm{NO}_{x}, \mathrm{VOCs}$ and $\mathrm{PM}_{10}$ emissions in the GMR;

- A list of identified emission abatement initiatives;

- Assessment of each initiative to determine important costing information and emissions abatement likely to be achieved;

- Cost curves for $\mathrm{NO}_{\mathrm{x}}, \mathrm{VOCs}$ and $\mathrm{PM}_{10}$; and

- Optimisation of the curves to consider the multi-pollutant framework of emissions abatement. 


\section{Reference case emissions}

The reference case used for the MACC modelling represents emissions for the GMR (Sydney, Newcastle, Wollongong and Non Urban sub regions), projected from 2003 to 2031 [2]. The base case assumed that prior trends in emissions profiles and drivers continue as they have previously, adjusted for existing or highly likely policies or industry projects. In this sense, the reference case shows future emissions profiles excluding any abatement efforts beyond those reasonably expected through business as usual. The reference case was based on the emission projections which were developed for the NSW Air Emissions Inventory (2003 base year) and which forecast emissions in five anthropogenic sectors: Industrial, Commercial, Domestic-Commercial, On-Road Mobile and Non-Road Mobile.

\section{MACC modelling methodology}

The methodology involved sequential and complementary steps, as follows:

- The construction of a reference case for abatement of NOX,VOCs and PM10 in the GMR, based on the emissions projections developed for the NSW Air Emissions Inventory 2003;

- The identification of priority emission sources with reference to NSW Air Emissions Inventory 2003, ranked by total mass of NOX, VOCs and PM10 emissions;

- The identification of abatement options, costs and the magnitude of emission reductions for single and multiple pollutants, available from relevant actions, to deliver reductions in anthropogenic emissions of NOX, VOCs and PM10 in the NSW GMR;

- The development of the MACC model $[3,4]$ that provided spreadsheets which graphically present incremental and total abatement costs and emission reductions for each pollutant; and

- The construction of marginal abatement cost curves using the multipollutant framework that accounts for interdependencies between pollutants and across sectors.

The methodology was informed by a review of integrated pollution management programs and models developed by international agencies.

\section{Abatement initiatives}

Separate pollutant MACCs were developed for the GMR, Sydney and Wollongong. A total of 35 abatement initiatives were developed for the GMR with a smaller subset of these applicable in the Sydney and Wollongong regions. A summary of the abatement initiatives identified and the pollutants affected by the measure are shown in Table 1. 
Table 1: $\quad$ Emissions abatement of initiatives.

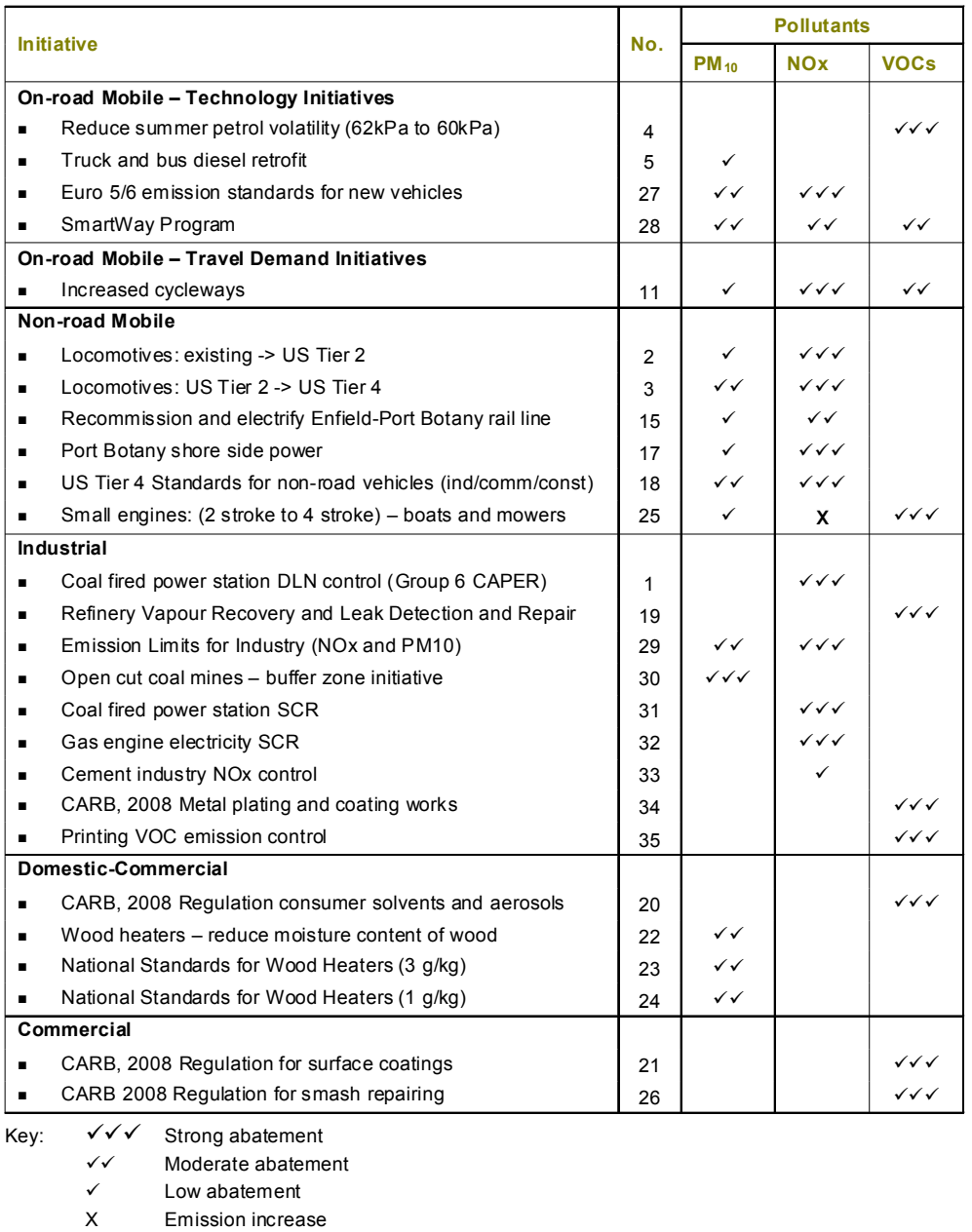

Table 2 represents the \% reduction from 2003 levels in regional emissions that the study estimated could be abated through the full implementation of all initiatives:

Table 2: $\quad$ Identified potential emission reductions.

\begin{tabular}{|lccc|}
\hline \multicolumn{4}{|c|}{ Maximum Identified Reductions of 2003 Emissions } \\
\hline Region & NOx & $\mathrm{PM}_{10}$ & VOC \\
\hline GMR & $58 \%$ & $25 \%$ & $18 \%$ \\
Sydney & $27 \%$ & $10 \%$ & $18 \%$ \\
Wollongong & $8 \%$ & $4 \%$ & $17 \%$ \\
\hline
\end{tabular}


From its analysis, SKM has been able to generate three separate single pollutant MACCs, showing the relative cost and size of potential abatement for each of the identified initiatives. Because it is problematic to allocate costs to one pollutant or another, SKM initially constructed the three individual MACCs as if each pollutant was the only one being considered, and bears the full cost of each initiative (even where that initiative reduces other pollutants). Reconciliation of costs and abatement of all three pollutants was then conducted in a subsequent optimisation phase. Figure 1 shows the MACC for NOx in Sydney.

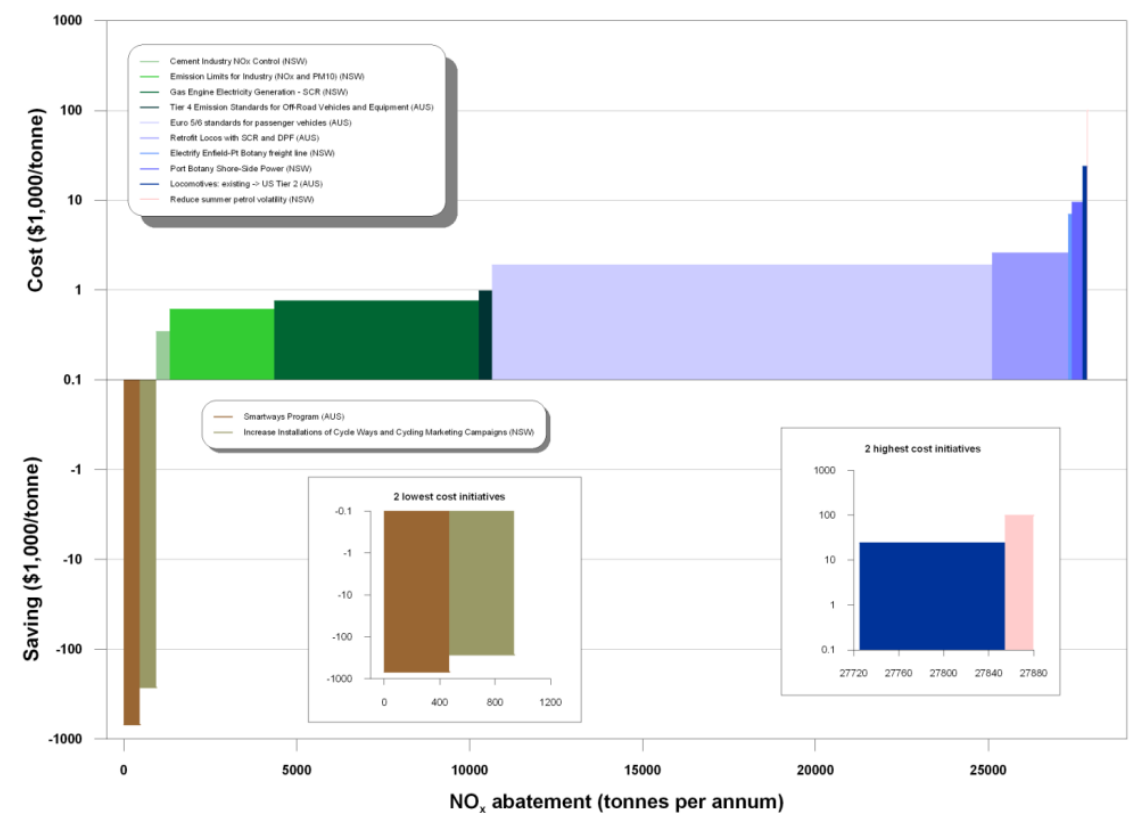

Figure 1: $\quad$ Sydney GMR $\mathrm{NO}_{\mathrm{X}}$ MACC.

Note that the MACC curves can include "negative abatement" (an increase in substance emissions) because of interactions between the three substances. A single-substance MACC study would normally exclude initiatives that would increase emissions of that substance. In a multi-substance MACC study, an initiative may abate one substance while increasing emissions of another substance. SKM developed an approach that allows "negative abatement" to be captured in the MACC developed for each substance and considered in the final multi-substance optimisation stage.

Some travel demand management measures identified, which were directed primarily to other benefits (such as public transport provision) and for which air emission reductions are only an ancillary benefit, were not considered in the final report. These appeared excessively costly when considered in the context of emission reductions only and were not genuinely comparable to other actions specifically designed to reduce emissions. 
In reviewing the MACC results, SKM found that while the optimal mix of initiatives differs for each pollutant, there is a significant degree of overlap in initiatives. That is, the analysis showed a number of initiatives that will have multiple benefits.

There are many significant results that can be drawn from the MACC modelling as shown in the MACC charts. Some key results follow.

\subsection{GMR}

NOX reduction in the GMR is dominated by two initiatives to reduce emissions from coal fired power stations being Dry Low NOX (DLN) and Selective Catalytic Reduction (SCR). These initiatives can effectively be considered mutually exclusive - with the option chosen for implementation depending on the total level of abatement required (DLN for lower levels up to around $15 \%$ abatement, and SCR for levels between around $15 \%$ and $30 \%$ ).

PM10 reduction is dominated by the effect of buffer zones of future open cut coal mines being equated to emission reductions. The costs associated with the modelled measure are provided as a guide to the investment in land and property required to mitigate adverse impacts by providing buffer zones to separate future mine developments and sensitive receivers. Recognising the importance of particle emissions from coal, $\mathrm{OEH}$ subsequently commissioned a separate study to identify further measures to reduce coal mine emissions (OEH 2010).

Options were identified for the GMR to reduce both NOX and PM10 by the indicative $25 \%$ target identified for this study, though initiatives identified only reduce VOC by $18 \%$.

\subsection{Sydney}

The profile of abatement options in the Sydney region is similar to the GMR. However, with no coal power stations or open cut coal mines in the Sydney region, the largest NOX and PM10 measures identified for the GMR were not applicable within the Sydney region.

The study indicated that NOX emission reductions in the Sydney region need to focus on motor vehicle emissions and specifically the introduction of emission standards for motor vehicles, such as Euro 5/6 standards for passenger cars and light commercial vehicles.

Introduction of Californian Air Resources Board (CARB) emission standards for VOC sources, in particular surface coatings and consumer products, have the potential to reduce significant quantities of VOC emissions.

Increased regulation of wood heaters in line with proposed national standards has the potential to reduce the greatest amounts of PM10 in Sydney.

Reduction of NOX by greater than $25 \%$ would be possible, but reductions of only $10 \%$ of PM10 and 18\% of VOC were identified for Sydney.

\subsection{Wollongong}

Aside from the Port Kembla Steelworks, which were not specifically considered by the study, emission reductions of NOX, VOC and PM10 in Wollongong 
could be achieved by similar initiatives to those identified for Sydney.

As per the Sydney region, NOX abatement is dominated by Euro 5/6 standards for vehicles, PM10 abatement by low emission standards for wood heaters, and VOC abatement by CARB standards and small engine (boat and lawnmower) emission standards.

Lower proportional abatement potential in the Wollongong region was identified, amounting to $8 \%$ of NOx, $4 \%$ of PM10, and $17 \%$ of VOC. To achieve even moderate reductions in the Wollongong region would require virtually all applicable initiatives to be implemented.

\section{Optimised mix of initiatives}

Besides the individual MACCs for each substance, SKM used Linear Programming to solve the multi substance optimisation, and used the results to analyse the optimum mix of initiatives to meet a notional $25 \%$ reduction in NOX and VOCs and $10-30 \%$ for PM10.

The list of initiatives in approximate priority order, based on the optimum mix table above, is shown in Table 3.

Note that the model gives priority to negative or lower cost initiatives, although they may deliver only a small percentage of the emission reductions required.

Because some initiatives reduce more than one substance, and in some instances even increase emissions of one substance while reducing another, determining the optimum mix can be complex, and is not as simple as taking the lowest cost measures from each of the three individual MACCs.

SKM's optimisation approach relied on a linear programming mathematical method to solve a function that minimises the total cost of all abatement, while achieving three constraints requiring the abatement of each of the three substances to at least equal the target amount. The method used an iterative approach to arrive at an optimum least cost solution.

Note that the full $25 \%$ target abatement was not achievable for all substances with the programs identified. The actual level of abatement varied by substance and region.

A mix of relatively few initiatives is required to achieve abatement levels up to around $15 \%$. Above that, the majority of cost effective initiatives identified are required. From a policy perspective it is noteworthy that an abatement initiative selected as a cost effective measure to meet a given target may no longer be cost effective when the abatement target is altered. The "end point" must be known when considering the mix of policy options. Gradually rolling out initiatives in order moving up the abatement curve may not result in the optimal mix at higher abatement levels.

Where options were mutually exclusive options, SKM selected the option that would provide the least cost abatement across the largest range of abatement. 
Table 3: $\quad$ Priority measures to abate emissions.

\begin{tabular}{|c|c|}
\hline Priority & GMR \\
\hline High & $\begin{array}{l}\text { - } \quad \text { Shift Transport Mode to Cycling * } \\
\text { - } \quad \text { SmartWay Program * } \\
\text { - } \quad \text { Peal Fired Power Station NOx Control - Low NOx Burners } \\
\text { - } \quad \text { CARB } 2008 \text { Regulation for Surface Coatings - Architectural_Industrial_Maintenance (AIM) } \\
\text { - } \quad \text { Small engines: (2 stroke to } 4 \text { stroke) Recreational Boating and Lawn Mowing } \\
\text { - } \quad \text { Metal Plating and Coating Works: CARB, } 2008 \text { AIM Regulation } \\
\text { - } \quad \text { Printing VOC Emissions Control } \\
\text { - } \quad \text { Euro } 5 / 6 \text { Emission Standards for New Passenger Vehicles }\end{array}$ \\
\hline Medium & - Summer-time Petrol Volatility (62 kPA to $60 \mathrm{kPA}$ ) \\
\hline \multirow[t]{2}{*}{ Low } & - $\quad$ Tier 2 -> Tier 4Locomotives: Selective Catalytic Reduction and Diesel Particulate Filter \\
\hline & Measures requiring higher priority for Sydney region (in addition to those identified above) \\
\hline \multirow[t]{2}{*}{ High } & $\begin{array}{ll}\text { - } & \text { Diesel Locomotive Replacement USEPA Tier } 0 \text {---> Tier } 2 \\
\text { - } & \text { Retrofit Tier } 2 \text { Locomotives with Selective Catalytic Reduction (SCR) and Diesel Particulate } \\
& \text { Filter (DPF) } \\
\text { - } & \text { Truck and Bus Diesel Retrofit } \\
\text { - } & \text { Tier } 4 \text { Emission Standards for Off-Road Vehicles and Equipment (Industrial) and } \\
& \text { (Commercial and Construction) } \\
\text { - } \quad \text { Wood Heaters - Reduce the Moisture Content of Firewood } \\
\text { - National Standards for Wood Heaters }(3 \mathrm{~g} / \mathrm{kg}) \\
\text { - National Standards for Wood Heaters }(1 \mathrm{~g} / \mathrm{kg}) \\
\text { - } \quad \text { Emission Limits for Industry (NOx and PM10) } \\
\text { - } \quad \text { Gas Engine Electricity Generation - SCR } \\
\text { - } \quad \text { Cement Industry NOx Control }\end{array}$ \\
\hline & $\begin{array}{l}\text { Measures requiring higher priority for Wollongong region (in addition to those identified } \\
\text { above) }\end{array}$ \\
\hline High & $\begin{array}{l}\text { - } \quad \text { Diesel Locomotive Replacement USEPA Tier } 0 \text {---> Tier } 2 \\
\text { - } \quad \text { Truck and Bus Diesel Retrofit } \\
\text { - } \quad \text { Tier } 4 \text { Emission Standards for Off-Road Vehicles and Equipment (Industrial) and } \\
\text { (Commercial and Construction) } \\
\text { - Wood Heaters - Reduce the Moisture Content of Firewood } \\
\text { - National Standards for Wood Heaters }(3 \mathrm{~g} / \mathrm{kg}) \\
\text { - } \quad \text { Euro } 5 / 6 \text { Emission Standards for New Passenger Vehicles }\end{array}$ \\
\hline
\end{tabular}

\subsection{Optimised abatement for the GMR}

Figure 2 shows the results of SKM's optimisation analysis for the GMR. Note that maximum achievable VOC abatement was $18 \%$, so, with the identified measures, the full solution of 25\% reduction in NOX and VOCs and 10-30\% for PM10 could not be found. For clarity, the options with net benefits have been excluded from the cost analysis, as they can be implemented as "no regrets" options. Their strongly negative costs distort the impact of other initiatives. As they exhibit relatively small abatement (between $0.1 \%-0.3 \%$ ) compared to total identified abatements, their omission does not materially alter the results.

Figure 2 shows that VOC is the primary driver of costs, with the cost of achieving comparable concurrent abatement in other substances only minimally more expensive than VOC alone.

Abatement up to around $15 \%$ of all three substances can be achieved at a relatively low net present cost (over 20 years) of around $\$ 717$ million, with a sharp increase in costs for abatement above $15 \%$. The total net present cost of the optimised suite of abatement initiatives for the GMR, to achieve reductions in NOX, VOCs and PM10 of 25\%, 18\% and 25\% respectively, would be $\$ 1.45$ billion. 


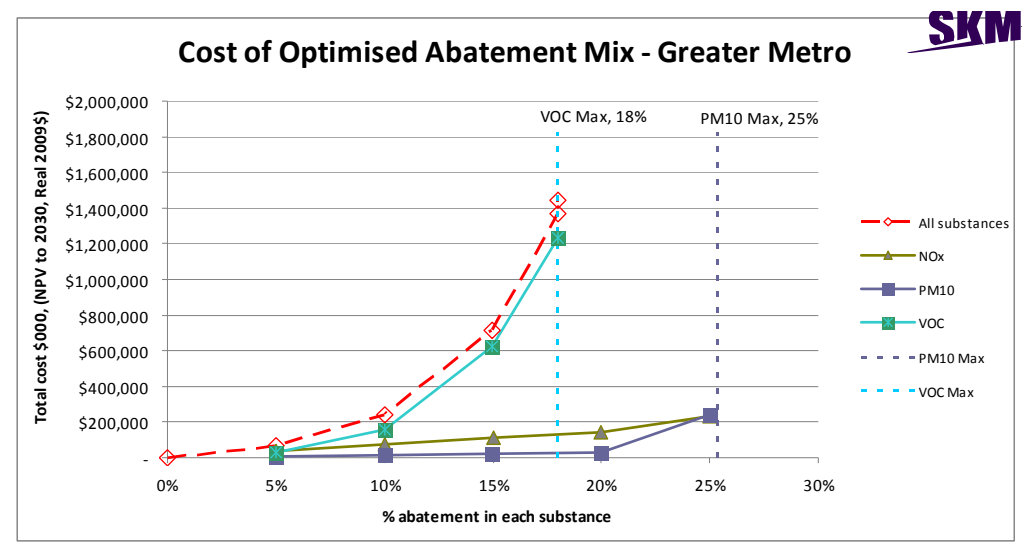

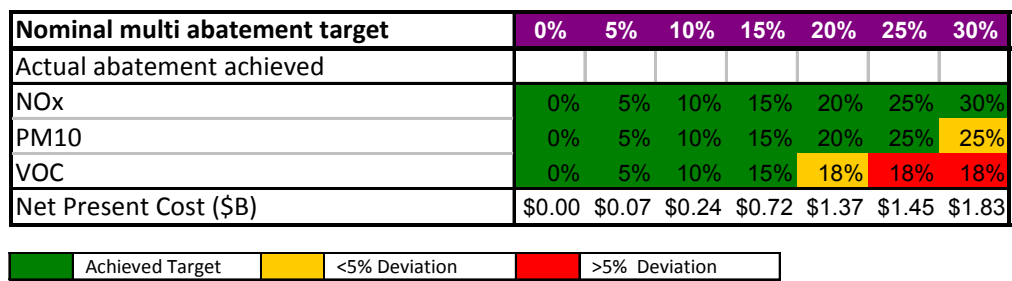

Figure 2: $\quad$ Optimised abatement for the NSW GMR.

Greater abatement percentages for NOX and PM10 could be achieved at lower cost, indicating a differentiated target for VOC of around $15 \%$ could be a lower cost policy option. However, considerations other than costs, in particular interactions between NOx and VOCs, are critical to setting VOC emission reduction targets. SKM did not have sufficient information to assess an alternative VOC target.

\subsection{Optimised abatement Sydney}

Figure 3 shows the optimised cost of abatement for the Sydney region.

The chart shows that the cost of achieving concurrent percentage abatement up until $10 \%$ for all three substances is only minimally more expensive than for PM10 alone. For further abatement of, between $10 \%$ and $20 \%$, cost of abatement of VOC is the primary driver of cost (holding PM10 abatement constant at 10\%). As the Figure 3 chart and table show, concurrent abatement of all substances to $25 \%$ could not be achieved, as identified initiatives for PM10 and VOC were insufficient to achieve $25 \%$ abatement.

The maximum abatement potential identified is $27 \%$ for NOX, $18 \%$ for VOC and $10 \%$ for PM10, which is estimated to cost (net present value) a total of $\$ 1.27$ billion, with a sharp increase in costs at around 10\% "multi abatement" due to PM10 measures having been exhausted and the more expensive VOC measures 


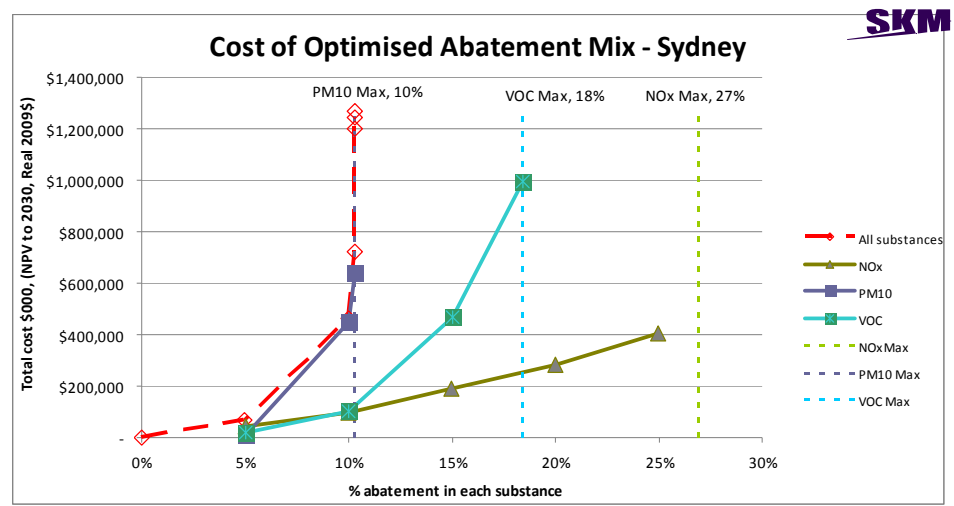

\begin{tabular}{|c|c|c|c|c|c|c|c|}
\hline Nominal multi abatement target & $0 \%$ & $5 \%$ & $10 \%$ & $15 \%$ & $20 \%$ & $25 \%$ & $30 \%$ \\
\hline \multicolumn{8}{|l|}{ Actual abatement achieved } \\
\hline NOx & $0 \%$ & $5 \%$ & $18 \%$ & $21 \%$ & $21 \%$ & $25 \%$ & $27 \%$ \\
\hline PM10 & $0 \%$ & $5 \%$ & $10 \%$ & $10 \%$ & $10 \%$ & $10 \%$ & $10 \%$ \\
\hline VOC & $0 \%$ & $5 \%$ & $10 \%$ & $15 \%$ & $18 \%$ & $18 \%$ & $18 \%$ \\
\hline Net Present Cost (\$B) & $\$ 0.00$ & $\$ 0.07$ & $\$ 0.47$ & $\$ 0.72$ & $\$ 1.20$ & $\$ 1.24$ & $\$ 1.27$ \\
\hline
\end{tabular}

\begin{tabular}{|l|l|l|l|l|l|l|}
\hline Achieved Target & & Exceeded Target & & $\leq 5 \%$ Deviation & & $>5 \%$ Deviation \\
\hline
\end{tabular}

Figure 3: $\quad$ Optimised abatement for Sydney.

being implemented. The nominal 25\% abatement target point results in $25 \%$ abatement for NOX, $18 \%$ for VOC, and 10\% for PM10, which could be achieved at a total net present cost of $\$ 1.24$ billion. A $10 \%$ abatement of all three pollutants could be achieved at a net present cost of around $\$ 465$ million. Some differentiation of targets for the three substances could achieve close to the maximum potential abatement at reduced cost.

Again, SKM did not assess the economic benefits of lower VOC emission reduction targets. The MACCs and optimisation results provide input to further modelling of ambient air quality by $\mathrm{OEH}$ and further policy analysis. Conclusions

The SKM study of measures to reduce air emission in NSW identified and analysed a range of potential abatement initiatives across the NSW GMR. SKM developed MACCs for NOx, VOC and PM10 in the NSW GMR and sub regions. An optimisation model was developed that assessed the MACCs in terms of economic, environmental and social impacts as well as technical feasibility.

A total of 35 abatement initiatives were developed for the GMR with a smaller subset of these applicable in the Sydney and Wollongong regions. The resulting curves identified potential sets of strategies that could be applied to reduce emissions, although the measures modelled were insufficient to achieve target emission reductions of VOC and PM10 in some regions.

The MACCs provide a guide to prioritising potential actions for further investigation. Further full analysis of potential actions is required to determine 
the actual costs and emission abatement potential of the actions identified, as well as other benefits that may contribute to a program's value.

The Linear Programming optimisation model identified a multi substance optimum mix of initiatives to meet a notional $25 \%$ reduction in NOX and VOCs and $10-30 \%$ for PM10. Because some initiatives act on more than one air pollutant, in some instances an increase in emissions of one substance occurred while reducing another.

Strategies to abate up to around $15 \%$ of all three substances in the GMR were identified that could nominally be achieved at relatively low net present cost (over 20 years), of around $\$ 717$ million. A sharp increase in costs for abatement was found when greater than $15 \%$ abatements of NOx, VOC and PM10 were sought. The total net present cost of the optimised suite of abatement initiatives for the GMR, to achieve reductions in NOx, VOCs and PM10 of $25 \%, 18 \%$ and $25 \%$ respectively, was estimated to be $\$ 1.45$ billion, noting that the costs are preliminary and more detailed estimates would be needed to assess the implementation of any measures.

\section{References}

[1] DECC, 2007, Current and projected air quality in NSW, A technical paper supporting the Clean Air Forum 2007, Department of Environment and Climate Change, NSW, Sydney.

[2] DECC 2008, Air Emissions Inventory for the Greater Metropolitan Region in New South Wales, Ozone Forming Potential for all Sectors: Results, Department of Environment and Climate Change (DECC) NSW, Sydney, NSW 2000, Australia.

[3] Molsener and Requate, 2007, 'Optimal abatement in dynamic multipollutant problems when pollutants can be complements or substitutes' Journal of Economic Dynamics and Control, vol. 31, pp. 2293-2316.

[4] Socolow, R., Pacala, S. and Greenblatt, J. 2004, Wedges: early mitigation with familiar technology, Princeton University, Princeton NJ 08544. 